Bond University

Research Repository

\title{
Comparing measures of fat-free mass in overweight older adults using three different bioelectrical impedance devices and three prediction equations
}

\author{
Ramsey, Rebecca; Isenring, E; Daniels, L \\ Published in: \\ Journal of Nutrition, Health and Aging
}

DOI:

$10.1007 / \mathrm{s} 12603-011-0085-6$

\section{Licence:}

Other

Link to output in Bond University research repository.

Recommended citation(APA):

Ramsey, R., Isenring, E., \& Daniels, L. (2012). Comparing measures of fat-free mass in overweight older adults using three different bioelectrical impedance devices and three prediction equations. Journal of Nutrition, Health and Aging, 16(1), 26-30. https://doi.org/10.1007/s12603-011-0085-6

\section{General rights}

Copyright and moral rights for the publications made accessible in the public portal are retained by the authors and/or other copyright owners and it is a condition of accessing publications that users recognise and abide by the legal requirements associated with these rights.

For more information, or if you believe that this document breaches copyright, please contact the Bond University research repository coordinator 
Title: Comparing measures of fat-free mass in overweight older adults using three different bioelectrical impedance devices and three prediction equations.

Running title: Body composition by three BIA devices

Rebecca Ramsey ${ }^{1}$, Elisabeth Isenring ${ }^{1}$, Lynne Daniels ${ }^{1}$

${ }^{1}$ Queensland University of Technology, Brisbane, Queensland 4059 Australia

RR was the primary data collector and main author of the manuscript and assisted in statistical analysis and interpretation as part of the requirements for a Bachelor of Health Science (Honours). EI initiated and supervised the project and assisted in data analysis and interpretation and in writing the manuscript. LD initiated and supervised the project and assisted in writing the manuscript.

Correspondence: All correspondence in regarding this article can be sent to:

Rebecca Ramsey

School of Public Health - Institute of Health and Biomedical Innovation

Queensland University of Technology

Victoria Park Road, Kelvin Grove, Brisbane, Queensland 4059

Phone: (07) 31388289

Fax: (07) 31383130

E-mail: rebecca.ramsey@qut.edu.au

Key words: Body composition, bioelectrical impedance analysis (BIA), bioelectrical impedance spectroscopy (BIS), older adults 
ABSTRACT:

Objectives: To compare measures of fat-free mass (FFM) by three different bioelectrical impedance analysis (BIA) devices and to assess the agreement between three different equations validated in older adult and/or overweight populations.

Design: Cross-sectional study.

Setting: Orthopaedics ward of Brisbane public hospital, Australia.

Participants: Twenty-two overweight, older Australians (72 yr \pm 6.4, BMI $34 \mathrm{~kg} / \mathrm{m}^{2}$ $\pm 5.5)$ with knee osteoarthritis.

Measurements: Body composition was measured using three BIA devices: Tanita 300-GS (foot-to-foot), Impedimed DF50 (hand-to-foot) and Impedimed SFB7 (bioelectrical impedance spectroscopy (BIS)). Three equations for predicting FFM were selected based on their ability to be applied to an older adult and/ or overweight population. Impedance values were extracted from the hand-to-foot BIA device and included in the equations to estimate FFM.

Results: The mean FFM measured by BIS $(57.6 \mathrm{~kg} \pm 9.1)$ differed significantly from those measured by foot-to-foot $(54.6 \mathrm{~kg} \pm 8.7)$ and hand-to-foot BIA $(53.2 \mathrm{~kg} \pm 10.5)$ $(\mathrm{P}<0.001)$. The mean \pm SD FFM predicted by three equations using raw data from hand-to-foot BIA were $54.7 \mathrm{~kg} \pm 8.9,54.7 \mathrm{~kg} \pm 7.9$ and $52.9 \mathrm{~kg} \pm 11.05$ respectively. These results did not differ from the FFM predicted by the hand-to-foot device ( $\mathrm{F}=$ $2.66, \mathrm{P}=0.118)$.

Conclusions: Our results suggest that foot-to-foot and hand-to-foot BIA may be used interchangeably in overweight older adults at the group level but due to the large limits of agreement may lead to unacceptable error in individuals. There was no difference between the three prediction equations however these results should be confirmed within a larger sample and against a reference standard. 


\section{Introduction}

Physiological changes to body composition, including a decrease in fat-free mass (FFM) and a relative or actual increase in fat mass (FM), occur with aging and are associated with higher risk of morbidity and mortality [1-5]. Bioelectrical impedance analysis (BIA) is a simple and non-invasive means of estimating total body water (TBW), which is used to measure FFM and consequently FM, to assess the impact of disease and/or medical and nutritional interventions $[4,6]$. Traditional singlefrequency BIA is based on hand-to-foot measurement and involves the application of electrodes to the wrists and ankles of the body in the supine position, through which a small electrical current is transferred. In recent years, foot-to-foot BIA devices have become cheaper and more widely available $[4,6]$. Foot-to-foot analysis requires the user to stand upright on the foot-plates of the device, through which the electrical current is transferred. Foot-to-foot measurement may be advantageous as it is easier to use in both clinical and research settings, however there exist concerns as to the accuracy of measurement [7]. For both hand-to-foot and foot-to-foot BIA, the impedance to the current may be entered into empirical linear regression equations which estimate TBW, FM and/or FFM [4, 6, 8-9]. These equations must be reevaluated when applied to populations other than that in which they were developed [10]. Alternatively, the impedance to the current generated during BIA may be entered into pre-programmed equations within the device for immediate generation of results for FFM. Devices allowing bioelectrical impedance spectroscopy (BIS) to be performed are also available. The difference between BIS and BIA lies in the fact that BIS screens across a wider range of frequencies and utilises mathematical modelling and mixture equations (Cole-Cole plot and Hanai formula) to measure the difference between the resistance of the TBW and the resistance of the extracellular fluid (ECW) [11]

As BIA and BIS devices become more readily available, and their use more widespread, it is important to determine the comparability of the results generated by different types of devices. Few studies have compared measurements between different BIA and BIS devices, thus it is unknown whether devices can be used interchangeably in a clinical setting. Also, there are few studies which compare the 
results from BIA devices to those from published prediction equations [12-14] that have been previously derived and validated within older populations. Clinical recommendations for the use of BIA suggests that routine assessment should be undertaken only among healthy patients or those with a stable water and electrolyte balance, using a validated equation or device setting appropriate for the individual's age, gender and ethnicity [6]. Commercial BIA devices include pre-programmed equations designed for use in particular populations, including athletes, children, healthy adults and obese adults (up to $34 \mathrm{~kg} / \mathrm{m}^{2}$ ). The accuracy of BIA and BIS devices is dependent on hydration status, as altered hydration may compromise body conductivity [11]. As such, BIA and BIS have only been shown to be valid up to a BMI of $34 \mathrm{~kg} / \mathrm{m}^{2}$ [11] and routine assessment among obese individuals or those with unstable hydration status is not recommended without further validation of appropriate equations [6]. It remains unknown as to whether these pre-programmed equations are suitable for use among an overweight older adult population, as both obesity and aging may result in altered hydration status, body water distribution and possible FFM overestimation [11, 15]. Furthermore, aging is associated with other significant co-morbidities, such as impaired renal function, which may further alter hydration status [4].

The aims of this study were to: 1) compare measurements of FFM by traditional handto-foot BIA with measurements of FFM by foot-to-foot BIA and BIS; and 2) compare the FFM predicted by the pre-programmed equation from the traditional hand-to-foot BIA device to FFM predicted by three different prediction equations developed in older and/or overweight adults [12-14].

\section{Subjects and methods}

This study formed part of a pilot study investigating the benefits of weight reduction for older adults with knee osteoarthritis. A cross-sectional observational study was conducted within the context of a randomised controlled pilot study, held at the Royal Brisbane and Women's hospital (RBWH), investigating the effects of a lifestyle program compared to standard care in overweight patients with knee osteoarthritis. The study was approved by the university human research ethics committee 
(0700000220) and RBWH human research ethics committee (2007/034) and informed written consent was obtained from all participants. Criteria for inclusion in this study were overweight status $\left(\mathrm{BMI} \geq 27 \mathrm{~kg} / \mathrm{m}^{2}\right)$, age $\geq 60$ years, diagnosed knee osteoarthritis as per the American College of Rheumatology criteria for knee osteoarthritis and ambulatory with no use of walker, crutches or braces (the latter three criteria were to meet a subsequent aim of the project). Patients with current involvement in weight loss regimes, significant psychological distress or other barriers to participation or who were unable to understand or complete questionnaires were excluded from the study. Subjects were recruited from the previous surgery list, surgery waiting list and list of referrals from the Orthopaedics department of the RBWH. All patients who met the aforementioned selection criteria $(\mathrm{N}=188)$ were manually identified from these lists and invited to participate in the study via mail. Twenty-two individuals were eligible and agreed to participate in the study and complete data were obtained on 21 individuals. Prior to BIA analysis, height was measured to the nearest $0.1 \mathrm{~cm}$ using a wall-mounted stadiometer. Body composition was measured with three different BIA devices; the single-frequency foot-to-foot BIA (300GS Tanita Corporation of America Inc., IL, USA), single-frequency hand-to-foot BIA (ImpDF50, Impedimed, Australia) and BIS (Imp SFB7, Impedimed, Australia) which scans over a range of 256 different frequencies. Weight was measured to the nearest $0.1 \mathrm{~kg}$ (in clothing with no shoes) using the in-built scales of the foot-to-foot BIA. To ensure normal hydration status to optimise BIA capabilities and prevent errors in measurement due to fluid imbalance, subjects were requested to refrain from intensive physical activity and from consuming fluids 2 hours prior to measurement and from consuming alcohol 12 hours prior to measurement. After determining that participants did not have a pacemaker (of which function may be affected by the electrical current generated by a BIA device), participants were measured with an empty bladder and no jewellery. The same trained tester conducted the BIA measurements for all subjects. Foot-to-foot BIA: Foot-to-foot analyses contain two stainless steel foot-pad electrodes mounted on a platform scale; participants were required to stand upright and barefoot on the foot-to-foot device for measurements to be recorded. The standard adult setting was selected for measurement. Hand-to-foot BIA and BIS: Pre-gelled electrodes were applied to the hand, wrist, ankle and foot of 
the right-hand side of the body and measurement occurred with participants in a supine position; participants remained in this position for five minutes prior to measurement. The obese setting was selected for hand-to-foot BIA analysis [16]. BIS used new resistivity constants developed in 151 healthy adults (BMI $23.8 \pm 3 \mathrm{~kg} / \mathrm{m}^{2}$ [17]. Devices were checked for calibration on each day they were used.

Prediction equations that had been developed and validated in an older adult and/or an overweight population were identified from the literature and ranked according to their standard error of estimate (SEE). The equations were then selected (in descending order of SEE) if sufficient participant data were available for their use. Several equations were unsuitable as they required thigh circumference for use. Three prediction equations which differed from that pre-programmed into the hand-to-foot device [16] were selected from the literature (Table 1). Equation 1 was validated against dual x-ray absorptiometry (DXA) in 343 individuals aged between 22 and 94 years of age with BMIs ranging between $17 \mathrm{~kg} / \mathrm{m}^{2}$ (underweight) and $33.8 \mathrm{~kg} / \mathrm{m}^{2}$ (obese). This equation was selected as it was able to be applied to both overweight and older adult populations [12]. Equation 2 and Equation 3 were selected as they had been specifically validated among older populations [13-14] (Table 1). Equation 2 was validated against a four-compartment model (using TBW and total body potassium) among a wide range of BMIs (16.6 to $\left.33.8 \mathrm{~kg} / \mathrm{m}^{2}\right)$. Equation 3 was validated against DXA among a population with a mean BMI of $27.1 \mathrm{~kg} / \mathrm{m}^{2} \pm 4.6$ (women) and $28 \mathrm{~kg} / \mathrm{m}^{2} \pm 3.7(\mathrm{men})$; the BMI range was not specified. The raw impedance data from the hand-to-foot BIA device were then substituted into each equation for determination of FFM.

Statistical analysis was carried out using SPSS Version 15, 2007 (SPSS Inc., Chicago, IL, USA). A general linear model (repeated-measures ANOVA) was used to asses any significant difference in FFM estimated by the different BIA devices and FFM estimated by hand-to-foot BIA and the three prediction equations. Bland Altman (1986) analyses were used to compare results from the devices and the prediction equations to determine the bias and limits of agreement ( $\pm 2 \mathrm{SD}$ ) between the different devices and between results from the hand-to-foot device and the three prediction equations [18]. Correlation coefficients were also generated and compared 
between the three devices. Statistical significance was reported at $\mathrm{P}<0.05$ (two tailed).

\section{Results}

Twenty-two individuals (mean age $=72.0 \mathrm{yr} \pm 6.4$, mean BMI $=34.1 \mathrm{~kg} / \mathrm{m} 2 \pm 5.5,15$ obese: 7 overweight, $48 \%$ male) consented to participate in this study, with full data available on 21. The mean \pm SD of the FFM predicted by foot-to-foot BIA, hand-tofoot BIA, BIS and the published prediction equations are presented in Table 2.

The bias and limits of agreement for measurements between the two single-frequency devices are presented in Figure 1 and the bias, limits of agreement and correlation between the three devices are presented in Table 3. There were no statistically significant differences between the mean FFM estimated by the pre-programmed equations in the single-frequency hand-to-foot and foot-to-foot devices $(\mathrm{P}=0.140)$. However FFM measured by BIS differed significantly from both the hand-to-foot and the foot-to-foot devices $(\mathrm{P}<0.001)$. The bias, limits of agreement and correlation for differences between the pre-programmed equation in the hand-to-foot device and the prediction equations are presented in Table 4. As the assumption for sphericity was violated, the lower-bound equation was considered for comparison. There were no significant differences between the FFM measured by hand-to-foot BIA and the FFM predicted by the equations $(\mathrm{F}=2.66, \mathrm{P}=0.118)$.

\section{Discussion}

This study compared FFM as assessed by two BIA devices and a BIS device and compared FFM measured by the pre-programmed equation in the hand-to-foot BIA device with that predicted by three published equations applicable to older and/or overweight adults.

\section{Comparison of pre-programmed equations among three BIA devices}

The $1.4 \mathrm{~kg}$ difference in mean FFM between the hand-to-foot and foot-to-foot BIA devices was not statistically significant. However, differences in the FFM measured 
by BIS of $3 \mathrm{~kg}$ compared to the foot-to-foot devices and $4.4 \mathrm{~kg}$ compared to the handto-foot device were revealed to be statistically significant. These results combined with the strong, positive correlations $(r>0.9)$ suggest that the single frequency devices may be used interchangeably at the group level; however neither device could be used interchangeably with the BIS device which estimated a significantly higher mean FFM. A potential issue when comparing the foot-to-foot device with the handto-foot device was the fact that whilst the hand-to-foot had a specific obese setting, the foot-to-foot device only allowed the user to select from 'athlete' or 'normal'. Despite the differences in mode, the fact that there was no significant difference between the two devices suggests that these could be used interchangeably at the group level within this sample. Bland Altman analysis (Figure 1) demonstrated wide limits of agreement between the foot-to-foot and hand-to-foot devices, indicating that the FFM estimated by the foot-to-foot device may be $9.9 \mathrm{~kg}$ above or $7.1 \mathrm{~kg}$ below the FFM predicted by the hand-to-foot device. These large limits of agreement suggest the devices would not be appropriate for use at the individual level.

Comparison of pre-programmed equation in hand-to-foot BIA device with population-specific equations

There were no statistically significant differences between the FFM measured by the traditional hand-to-foot device and the FFM predicted by the three equations. The results produced by the hand-to-foot device were highly correlated with those produced by the equations $(r>0.9)$. This suggests that the obese equation in the handto-foot device is suitable for use in an overweight older adult population and can be used interchangeably with the prediction equations at the group level. Bland Altman analysis revealed wide limits of agreement between the results from the hand-to-foot and the results from the prediction equations, again suggesting that limitations in BIA exist at the individual level.

\section{Comparison with published literature}

Although our study focussed on a more obese sample than others, our results support previous findings, specifically those by Ritchie et. al (2005). Ritchie investigated the comparability of hand-to-foot and foot-to-foot BIA among fifty older adults aged 56 
to 94 years with a mean BMI of $28.7 \mathrm{~kg} / \mathrm{m}^{2} \pm 4.9$. The results showed a significant correlation between hand-to-foot and foot-to-foot BIA $(\mathrm{r}=0.84, \mathrm{P}<0.001)$ suggesting that devices could be used interchangeably, with the foot-to-foot device measuring a slightly higher mean FFM (36\%) than the hand-to-foot device $(35 \%)(\mathrm{P}=$ $0.13)$ [19].

In the current study, Bland Altman analysis revealed large limits of agreement suggesting that BIA may not be appropriate for use at the individual level. This supports findings by Isenring et. al (2004) who compared measures of TBW by footto-foot BIA with that predicted by a deuterium oxide dilution technique in 27 subjects undergoing outpatient radiotherapy (mean age $=62$ years \pm 15 , mean BMI $=$ $26.2 \mathrm{~kg} / \mathrm{m}^{2} \pm 3.6$ ). The study by Isenring et. al (2004) found that TBW predicted by foot-to-foot BIA could vary as much as $12 \mathrm{~L}$ above or $8.6 \mathrm{~L}$ below the actual TBW measured by a deuterium oxide dilution technique and thus may be limited at the individual level [20]. Our findings are also consistent with conclusions by Buchholz et. al, 2004, who undertook an extensive review of the literature and suggested that BIA was limited to the group level [4].

The major limitation of our study was that no reference standard was used with which to compare our measures for FFM. Consequently the true values for FFM are unknown. Previous studies comparing measurements in FFM between DXA, magnetic resonance imaging (MRI) and BIA have indicated that BIA tends to overestimate FFM compared to DXA [9-10, 21] and MRI [22]. Within our study, BIS predicted the highest mean FFM; this may suggest that BIS overestimates FFM. However, previous studies comparing BIS with the gold standard of isotope dilutions and reference standard of DXA contradict these findings. One such study by Moon et. al 2007 suggest that compared to deuterium oxide isotope dilution, BIS was a valid measure of total body water in young healthy adults $(r=0.98)$ [23]. Tengvall et. al (2008) also found that BIS was a valid measure of FFM in older adults $(\mathrm{N}=574)$, as there was no difference in FFM measure by BIS or DXA $(\mathrm{P}=0.58)$ [24]. The discrepancy between these results and our own may be explained by the characteristics of our study sample. As this study involved older adults participating 
in a weight loss trial we specifically targeted overweight and obese older adults. Consequently nearly $40 \%$ of our sample $(\mathrm{n}=8)$ exhibited a BMI of over $34 \mathrm{~kg} / \mathrm{m}^{2}$. BIA and BIS have only been shown to be valid up to a BMI of $34 \mathrm{~kg} / \mathrm{m}^{2}$ [11]. The disproportion between body mass and body conductivity that results from obesity as a result of altered hydration status and body water distribution may have resulted in overestimation of FFM in our sample [11, 15]. Furthermore, aging is associated with other significant co-morbidities (including liver disease, declining renal function which may also alter hydration status) [4]. Future studies should aim to derive resistivity constants specifically for overweight, older adult populations to determine whether this might further improve the use of BIS in measuring body composition in this population.

Although there was no statistically significant difference between the preprogrammed equation in the hand-to-food device the foot-to-foot BIA device and the three prediction equations from the literature, there appeared to be clinically relevant differences of $1.4 \mathrm{~kg}$ and $1.5 \mathrm{~kg}$ respectively (generally, professional opinion considers changes of $0.5-1 \mathrm{~kg}$ to be clinically significant) [25-27]. The relatively small size of our study sample means that these results should be confirmed within a larger sample.

In conclusion, these results suggest that foot-to-foot and hand-to-foot BIA devices may be used interchangeably at the group level, however cannot be used interchangeably with the BIS device in this sample of overweight adults. Due to the large limits of agreement, none of the BIA devices were suitable for use at the individual level. The findings of this study should be confirmed within a larger sample size and compared with a reference standard to determine the accuracy of measurement.

\section{Acknowledgements}

There were no conflicts of interest involved with this study. This work was supported by an Institute of Health and Biomedical Innovation (IHBI) Seeding grant. Rebecca Ramsey (RR) implemented the study as part of the requirements for a Bachelor of Health Science (Honours) and was the primary data collector and main author of the 
manuscript and assisted in statistical analysis and interpretation. Elisabeth Isenring was supported by a NHMRC Australian Clinical Training Fellowship (ID:324777) and contributed to study design, supervised data collection and contributed to data analysis, interpretation and manuscript preparation. Lynne Daniels designed the study, was the primary supervisor for RR and contributed to data interpretation and manuscript preparation. 


\section{References}

1. Ricciardi, R. and L.A. Talbot, Use of bioelectrical impedance analysis in the evaluation, treatment, and prevention of overweight and obesity. Journal of the American Academy of Nurse Practitioners, 2007. 19(5): p. 235-241.

2. Stallmann-Jorgensen, I.S., et al., General and visceral adiposity in black and white adolescents and their relation with reported physical activity and diet. International Journal of Obesity, 2007. 31(4): p. 622-629.

3. Remsberg, K.E. and R.M. Siervogel, A life span approach to cardiovascular disease risk and aging: the Fels Longitudinal Study. Mechanisms Of Ageing And Development, 2003. 124(3): p. 249-257.

4. Buchholz, A., C. Bartok, and D. Schoeller, The validity of bioelectrical impedance models in clinical populations. Nutrition in Clinical Practice, 2004. 19(5): p. 433-446.

5. Guida, B., et al., Bioelectrical impedance analysis and age-related differences of body composition in the elderly. Nutrition, Metabolism and Cardiovascular Diseases, 2007. 17: p. 175 - 180.

6. Kyle, U.G., et al., Bioelectrical impedance analysis-part II: utilization in clinical practice. Clinical Nutrition (Edinburgh, Scotland), 2004. 23(6): p. 1430-1453.

7. Lasser, S., et al., Evaluation of two foot-to-foot bioelectrical impedance analysers to assess body composition in overweight and obese adolescents. British Journal of Nutrition, 2003. 90: p. 987 - 992.

8. Demura, S., S. Sato, and T. Kitabayashi, Percentage of total body fat as estimated by three automatic bioelectrical impedance analyzers. Journal Of Physiological Anthropology And Applied Human Science, 2004. 23(3): p. 9399.

9. Barbosa-Silva, M.C.G. and A.s.J.D. Barros, Bioelectrical impedance analysis in clinical practice: a new perspective on its use beyond body composition equations. Current Opinion In Clinical Nutrition And Metabolic Care, 2005. 8(3): p. 311-317.

10. Heymsfield, S., et al., Techniques used in the measurement of body composition: an overview with emphasis on bioelectrical impedance analysis. American Journal of Clinical Nutrition, 1996. 64(3S): p. 478S-484.

11. Kyle, U.G., et al., Bioelectrical impedance analysis--part I: review of principles and methods. Clinical Nutrition (Edinburgh, Scotland), 2004. 23(5): p. 1226-1243.

12. Kyle, U.G., et al., Single prediction equation for bioelectrical impedance analysis in adults aged 20--94 years. Nutrition (Burbank, Los Angeles County, Calif.), 2001. 17(3): p. 248-253.

13. Roubenoff, R., et al., Application of Bioelectrical Impedance Analysis to Elderly Populations. The Journals of Gerontology, 1997. 52A(3): p. M129 M136.

14. Dey, D.K. and I. Bosaeus, Comparison of bioelectrical impedance prediction equations for fat-free mass in a population-based sample of 75 y olds:: The NORA study. Nutrition, 2003. 19(10): p. 858. 
15. Coppini, L., D. Waitzberg, and A. Campos, Limitations and validation of bioelectrical impedance analysis in morbidly obese patients. Current Opinion In Clinical Nutrition And Metabolic Care, 2005. 8: p. 329 - 332.

16. Segal, K.R., et al., Lean body mass estimation by bioelectrical impedance analysis: a four-site cross-validation study. American Journal of Clinical Nutrition, 1988. 47(7): p. 7 - 14.

17. Ward, L.C., et al. Constancy of resistivity coefficients for bioimpedance spectroscopy (BIS). in 8th International Symposium on In Vitro Body Composition Studies. 2008. New York, USA [Abstract].

18. Bland, J.M. and D.G. Altman, Statistical methods for assessing agreement between two methods of clinical measurement. Lancet, 1986. 1(8476): p. 307310.

19. Ritchie, J., C. Miller, and H. Smiciklas-Wright, Tanita Foot-to-Foot Bioelectrical Impedance Analysis System Validated in Older Adults. Journal of the American Dietetic Association, 2005. 105: p. 11617 - 11619.

20. Isenring, E., et al., Evaluation of foot-to-foot bioelectrical impedance analysis for the prediction of total body water in oncology outpatients receiving radiotherapy. European Journal of Clinical Nutrition, 2004. 58(1): p. 46-51.

21. Bolanowski, M. and B.E. Nilsson, Assessment of human body composition using dual-energy $x$-ray absorptiometry and bioelectrical impedance analysis. Medical Science Monitor: International Medical Journal Of Experimental And Clinical Research, 2001. 7(5): p. 1029-1033.

22. Varady, K.A., S. Santosa, and P.J.H. Jones, Validation of hand-held bioelectrical impedance analysis with magnetic resonance imaging for the assessment of body composition in overweight women. American Journal Of Human Biology: The Official Journal Of The Human Biology Council, 2007. 19(3): p. 429-433.

23. Moon, J., et al., Total body water estimations in healthy men and women using bioimpedance spectroscopy: a deuterium oxide comparison. Nutrition and Metabolism, 2008. 5(7).

24. Tengvall, M., et al., Body composition in the elderly: Reference values and bioelectrical impedance spectroscopy to predict total body skeletal muscle mass. Clinical Nutrition (Edinburgh, Scotland), 2008: p. 1 - 7.

25. Isenring, E., S. Capra, and J. Bauer, Nutrition intervention is beneficial in oncology outpatients receiving radiotherapy to the gastrointestinal or head and neck area. British Journal of Cancer, 2004. 91: p. 447 - 452.

26. Barber, M., et al., Fish oil enriched nutritional supplement attenuates progression of the acute-phase response in weight-losing patients with advanced pancreatic cancer. Journal of Nutrition, 1999a. 129: p. 1120 - 1125.

27. Barber, M., et al., The effect of an oral nutritional supplement enriched with fish oil on weight-loss in patients with pancreatic cancer. British Journal of Cancer, 1999b. 81: p. 80 - 86. 
Table 1: Characteristics of single frequency prediction equations for the prediction of Fat-free mass (FFM).

\begin{tabular}{|c|c|c|c|c|c|}
\hline Author & Population & $\mathbf{N}$ & Mean Age (yr) & $\begin{array}{l}\text { Mean BMI } \\
\left(\mathrm{kg} / \mathrm{m}^{2}\right)\end{array}$ & Equation \\
\hline \multirow[t]{2}{*}{$\begin{array}{l}\text { Segal et. al } \\
\text { Hand-to-foot* }\end{array}$} & $\begin{array}{l}\text { Adults } \\
17-62 \text { years }\end{array}$ & 1599 & $\mathrm{~W}: 35 \pm 9.0$ & Not specified & $\begin{array}{l}\mathrm{W}:\left(0.00091186 \times \mathrm{Ht}^{2}\right)-(0.01466 \\
\mathrm{x} \mathrm{R})+(0.2999 \times \mathrm{Wt})-(0.07012 \mathrm{x} \\
\text { age })+9.37938\end{array}$ \\
\hline & & & M: $32 \pm 9.0$ & Not specified & $\begin{array}{l}\text { M: }\left(0.000885 \times \mathrm{Ht}^{2}\right)-(0.02999 \times \mathrm{R}) \\
(0.42688 \times \mathrm{Wt})-(0.07002 \times \text { age }) \\
+14.52435\end{array}$ \\
\hline $\begin{array}{l}\text { Kyle et. al } \\
(2001)[12] \\
\text { Equation } 1\end{array}$ & $\begin{array}{l}\text { Adults } \\
20-94 \text { years }\end{array}$ & 343 & Not specified & $24.6 \pm 2.9$ & $\begin{array}{l}-4.104+\left(0.518 \mathrm{Ht}^{2} / \mathrm{R} 50\right)+(0.231 \mathrm{Wt}) \\
+(0.130 \mathrm{Xc})+(4.299 \mathrm{sex})\end{array}$ \\
\hline $\begin{array}{l}\text { Dey et. al } \\
\text { (2003) [14] } \\
\text { Equation } 2 \\
\end{array}$ & $\begin{array}{l}\text { Older adults } \\
70-75 \text { years }\end{array}$ & 823 & 71.9 & $26.2 \pm 3.7$ & $\begin{array}{l}11.78+0.499 \mathrm{ht}^{2} / \mathrm{R}_{50}+0.134 \mathrm{Wt} \\
+3.449 \mathrm{sex}\end{array}$ \\
\hline \multirow{2}{*}{$\begin{array}{l}\text { Roubenoff et. al } \\
(1997)[13] \\
\text { Equation } 3\end{array}$} & $\begin{array}{l}\text { Older adults } \\
60-95 \text { years }\end{array}$ & W: 294 & $\mathrm{~W}: 78.4 \pm 4.5$ & $\mathrm{~W}: 27.1 \pm 4.6$ & $\begin{array}{l}\mathrm{W}: 7.7435+0.4542 \mathrm{Ht}^{2} / \mathrm{R}_{50} \\
+0.1190 \mathrm{wt}+0.0455 \mathrm{Xc}\end{array}$ \\
\hline & & M: 161 & $M: 78.2 \pm 4.3$ & $M: 28.0 \pm 3.7$ & $\begin{array}{l}\mathrm{M}: 9.1536+0.4551 \mathrm{Ht}^{2} / \mathrm{R} 50 \\
+0.1926 \mathrm{wt}+0.0667 \mathrm{Xc}\end{array}$ \\
\hline
\end{tabular}

yr, years; BMI, body mass index; Ht, height (cm); Wt, weight; r, resistance; R50, resistance at $50 \mathrm{kHz}$; Xc, reactance; sex, gender where female $=0$, male $=1 ; \mathrm{W}$, women; $\mathrm{M}$, men.

* Pre-programmed in hand-to-foot device 
Table 2: Mean fat-free mass (FFM) measured by single-frequency hand-tofoot bioelectrical impedance analysis (BIA) device, single-frequency foot-tofoot BIA device, bioelectrical impedance spectroscopy (BIS) and three published prediction equations.

Device / equation mean FFM (kg) SD

Hand-to-foot 53.2

10.5

Foot-to-foot 54.6

BIS 57.6

Equation 1 [12] 54.7

Equation 2 [14] 54.7 7.9

Equation 3 [13] 52.9 11.1

kg, kilograms; SD, standard deviation.

Equation 1, prediction equation for FFM suitable for use in overweight/ obese adults [12].

Equation 2, prediction equation for FFM suitable for use in older adult populations [14]. Equation 3, prediction equation for FFM suitable for use in older adult populations [13]. 
Table 3: Bias and limits of agreements and correlation of fat-free mass (FFM) measured by two different bioelectrical impedance analysis (BIA) devices and bioelectrical impedance spectroscopy (BIS)

\begin{tabular}{lccccc}
\hline Device & $\begin{array}{c}\text { Comparison } \\
\text { device }\end{array}$ & $\begin{array}{c}\text { Bias } \\
(\mathbf{k g})^{* *}\end{array}$ & $\begin{array}{c}\text { Limits of } \\
\text { Agreement** } \\
\mathbf{( \pm 2 S D ) ( k g )}\end{array}$ & $\begin{array}{c}\text { Correlation } \\
(\mathbf{r})\end{array}$ & $\begin{array}{c}\mathbf{P} \\
\text { value* }\end{array}$ \\
\hline $\begin{array}{l}\text { Foot-to- } \\
\text { foot }\end{array}$ & Hand-to-foot & 1.43 & 8.5 & 0.92 & 0.140 \\
\hline $\begin{array}{l}\text { Foot-to- } \\
\text { foot }\end{array}$ & BIS & -3.01 & 7.1 & 0.92 & $<0.001$ \\
\hline $\begin{array}{l}\text { Hand- } \\
\text { to-foot }\end{array}$ & BIS & -4.44 & 7.4 & 0.94 & 0.001 \\
& & & & & \\
\hline
\end{tabular}

kg, kilograms

*P-value according to repeated measures ANOVA, sphericity assumed.

** Bias and limits of agreement determined by subtraction of FFM measured by 'comparison device' from FFM measured by 'device' 
Table 4: Bias and limits of agreements between fat-free mass (FFM) measured by preprogrammed equations in hand-to-foot bioelectrical impedance analysis (BIA) device and FFM predicted by single frequency prediction equations in an overweight or elderly population.

\begin{tabular}{llllc}
\hline Device & $\begin{array}{c}\text { Comparison } \\
\text { equation }\end{array}$ & $\begin{array}{c}\text { Bias } \\
\mathbf{( k g )}\end{array}$ & $\begin{array}{c}\text { Limits of } \\
\text { Agreement } \\
\mathbf{( \pm 2 S D ) ( k g )}\end{array}$ & $\begin{array}{c}\text { Correlation } \\
(\mathbf{r})\end{array}$ \\
\hline $\begin{array}{l}\text { Hand-to- } \\
\text { foot }\end{array}$ & Equation 1 [12] & -1.5 & 5.3 & 0.98 \\
\hline $\begin{array}{l}\text { Hand-to- } \\
\text { foot }\end{array}$ & Equation 2 [14] & -1.5 & 8.1 & 0.94 \\
\hline $\begin{array}{l}\text { Hand-to- } \\
\text { foot }\end{array}$ & Equation 3 [13] & -0.3 & 9.5 & 0.90 \\
\hline
\end{tabular}

$\mathrm{P}=0.118$ according to repeated measures ANOVA (lower-bound)

$\mathrm{kg}$, kilograms; SD, standard deviation.

Equation 1, prediction equation for FFM able suitable for use in overweight/ obese adults [12].

Equation 2, prediction equation for FFM suitable for use in older adult populations [14].

Equation 3, prediction equation for FFM suitable for use in older adult populations [13]. 


\section{Legend for Figures}

Figure 1: Bland Altman plot showing the difference in fat-free mass (FFM) measured by single frequency foot-to-foot bioelectrical impedance analysis (BIA) device and FFM measured by single frequency hand-to-foot device plotted against the mean difference in FFM 
Figure 1:

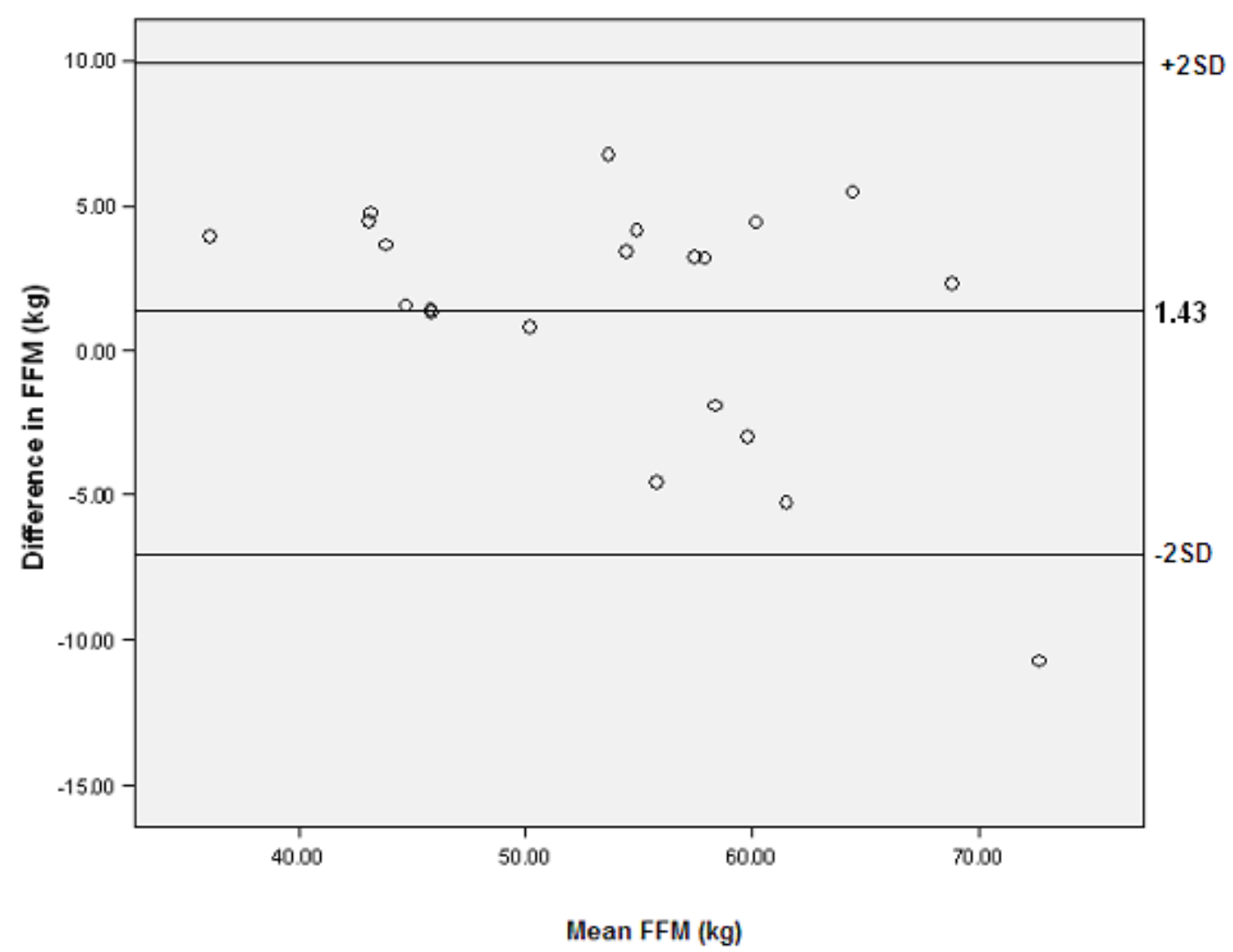

